\title{
Antibiotic Effects on Tenebrio molitor Ingestion of Styrofoam
}

\author{
Kieran Dunn \\ Boston College High School, 150 William T. Morrissey Boulevard, Boston, MA, 02125 USA; kn.dunn22@students.bchigh.edu
}

\begin{abstract}
Polystyrene is a significant environmental contaminant. Larvae of the mealworm, Tenebrio molitor, digest polystyrene, converting up to $49 \%$ of ingested polystyrene into carbon dioxide and the remainder into biomass. Microbiota in the mealworm gut help break down polystyrene, but little is known about the specific bacteria. This study estimates the microbial bacterial community makeup and size in the mealworm gut by sequencing the V4 region of the $16 \mathrm{~S}$ rRNA gene, the conserved gene in bacterial species. By modifying the gut microbiome with the administration of antibiotics, this study also attempts to determine how the microbiome is altered and whether such modifications are associated with changes in polystyrene digestion. Additionally, this study addresses whether an intake of polystyrene has adverse effects on the mealworm gut by looking for damage at the microscopic level and by measuring the antioxidants catalase and glutathione to assess for oxidative stress. Findings were that microbial diversity was key to maximal consumption of polystyrene. Suppression of microbiome diversity with antibiotics was associated with decreased polystyrene digestion. Polystyrene ingestion was associated with gut inflammation and damage, with decreased catalase levels and epithelial cell vacuolization.
\end{abstract}

KEYWORDS: Polystyrene; mealworms; Tenebrio molitor, 16s rRNA gene; microbiome; oxidative stress.

\section{- Introduction}

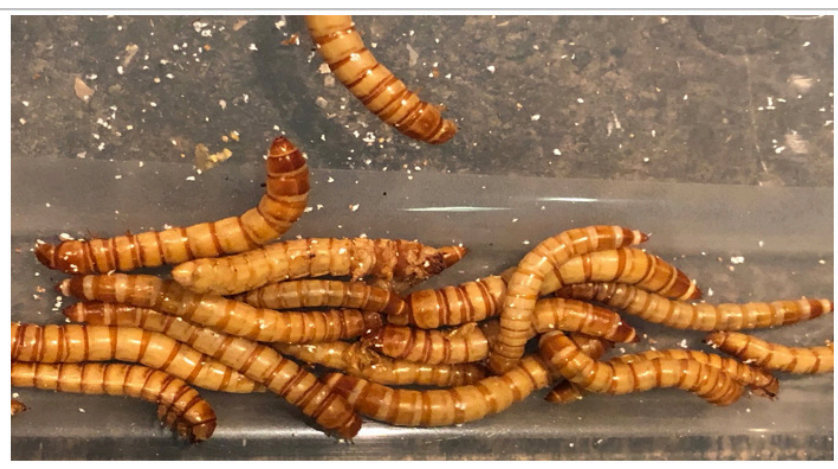

On Earth, we are contending with a major plastic problem, exemplified by the Great Pacific Garbage Patch. This is a floating island of garbage in the North Pacific Ocean which is twice the size of Texas and contains over 100,000,000 tons of plastic debris. ${ }^{1}$ The current global consumption of petroleum-based synthetic plastic is approximately 299 million tons per year. ${ }^{2}$ Global production of petroleum-based plastic has grown 200-fold from 1.5 million tons in 1950 to 299 million tons presently per year. ${ }^{3}$ Polystyrene (PS), molecular formula [-CH(C6H5)CH2-]n, commonly known as Styrofoam, accounts for approximately $7.1 \%$ (21 Mt/year) of the total plastic consumption.4 PS is generally considered non-biodegradable as a result of its high molecular weight and highly stable structure. ${ }^{2}$ However, PS products are often designed for a short service time and one-time use because of the low cost of this material. The sharp contrast between the remarkable durability and short service time of PS products has led to the increasing accumulation of PS waste in our environment. We need to find sustainable approaches in which we can reduce PS waste in environmentally responsible ways.
Larvae of the mealworm, Tenebrio molitor, digest polystyrene, converting up to $49 \%$ of ingested PS into carbon dioxide and the rest into biomass. ${ }^{2}$ Microbiota in the mealworm gut are responsible for breaking down the PS, but little is known about the specific bacteria within the gut, or the enzymes within these bacteria that digest PS. ${ }^{5}$ The mealworm gut can be considered an efficient bioreactor, or system supporting a biologically active environment. Physical and biochemical "treatment" (by chewing, ingesting, mixing, reacting with gut contents, and microbial degradation) are critical for the success of rapid PS degradation in the bioreactor. ${ }^{5}$ The mealworm gut microbiome may be adaptable, with different species of bacteria proliferating and enabling degradation of PS. ${ }^{5}$

This study focuses on estimating the microbial bacterial community makeup and size in the mealworm gut by sequencing the V4 region of the $16 \mathrm{~S}$ rRNA gene, the marker gene for bacterial species. ${ }^{6}$ The study also attempts to modify the gut microbiome with administration of various antibiotics, to determine how the microbiome is altered and whether such alterations are associated with changes in PS digestion. With growing concern regarding health effects of ingestion of plastic, this study also addresses effects on the mealworm gut during plastic ingestion, looking for evidence of oxidative stress or damage visualized histopathologically. ${ }^{7-9}$

Hypotheses for this study were two-fold: First, we hypothesize that the administration of antibiotics to mealworms alters the gut microbiome and decreases efficiency of Styrofoam ingestion. Second, we hypothesize that ingestion of Styrofoam is associated with the following gut inflammatory changes in the mealworm: histopathologic changes in the gut epithelium, and oxidative stress measured by decreased catalase and glutathione levels. 


\section{Results and Discussion Mealworm Mortality:}

The mealworm mortality did not vary markedly among experimental groups with an average survival of $73 \%$; this included surviving worms, worms that had developed into pupae, and sacrificed worms, $\mathrm{p}=0.99$ (Figure 1).

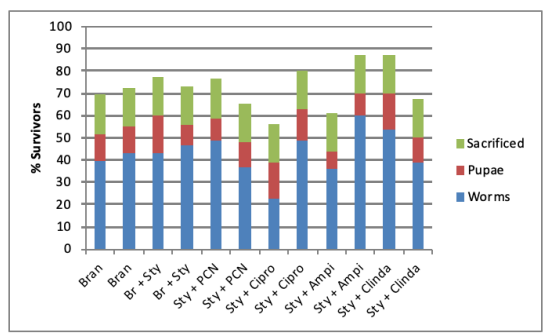

Figure 1: Antibiotic administration or Styrofoam exposure did not affect mealworm survival as measured by percent survivors at end of experimentation. $(\mathrm{Br}=$ bran, Sty $=$ Styrofoam, $\mathrm{PCN}=$ penicillin, Cipro $=$ ciprofloxacin, Ampi = Ampicillin, Clinda = Clindamycin.).

\section{Styrofoam Weight Loss:}

All groups of worms exposed to antibiotics digested less Styrofoam than worms fed Styrofoam alone (on average 73\% less by weight), $\mathrm{p}=0$. Worms exposed to Styrofoam alone consumed up to $9 \mathrm{~g}$ of Styrofoam (Figure 2). Worms exposed to bran + Styrofoam showed variable consumption of Styrofoam. One group appeared to prefer bran and ate little Styrofoam; the other group ate more Styrofoam than antibiotic-exposed worms, but less than worms fed Styrofoam alone. Surface area damage revealed a similar trend with bran + Styrofoam exhibiting maximal surface damage (11\%), and antibiotic exposure correlating with decreased surface damage, $\mathrm{p}=0$ (Figure 2).

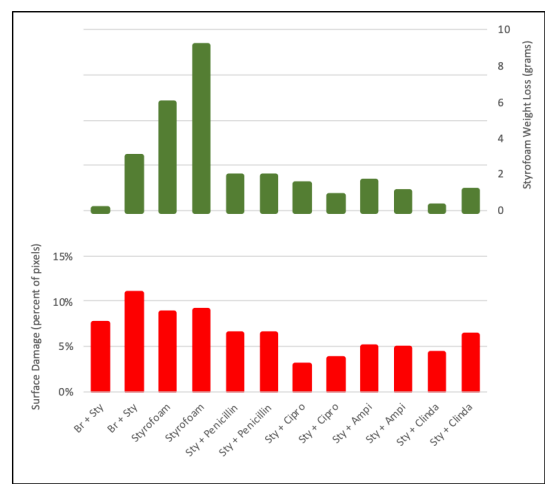

Figure 2: Antibiotic exposure was associated with decreased Styrofoam digestion as measured by Styrofoam weight loss and surface damage.

\section{Microbiome Testing:}

The microbial diversity in the mealworm gut was decreased with administration of antibiotics (Figure 3). Spiroplasmataceae was the most resistant bacteria to antibiotics and was present in most of the experimental groups. Enterobacteriaceae was the second most prevalent bacteria. Bacteria associated with maximal Styrofoam ingestion included a mixture of spiroplasmatacea, enterobacteriaceae, enterococcaceae, lactobacillaceae, flavobacteriaceae, veillonellaceae, moraxellaceae, leptotrichiaceae, pseudomonadaceae, cytophagaceae. There was a direct correlation between bacterial diversity and Styrofoam consumption, such that increased bacterial diversity was associated with increased consumption of Styrofoam, and decreased Styrofoam degradation occurred with decreased bacterial diversity. This direct correlation was most readily observed by superimposing relative abundance, weight loss and surface damage statistics in Figure 4.

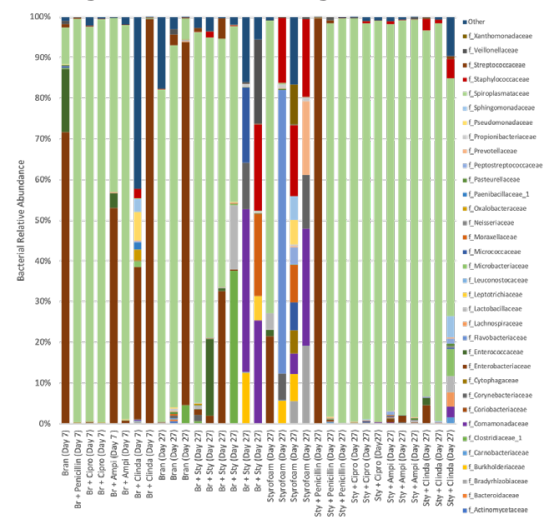

Figure 3: Decreased intestinal microbial diversity was observed with antibiotic exposure, as measured by relative abundance of bacterial families in intestinal samples.

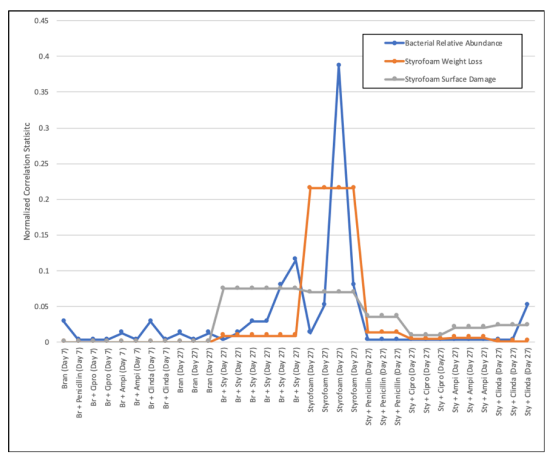

Figure 4: Styrofoam weight loss and surface damage are significantly correlated with intestinal bacterial relative abundance.

Table 1: Matrix K containing pairwise correlation coefficients and matrix $\mathrm{P}$ containing p-values for Pearson's correlation where bacterial relative abundance a, Styrofoam weight loss w, and Styrofoam surface damage s are correlated. Since the off-diagonal elements (cross correlations) of the $P$ matrix are less than 0.05 , the correlation statistic is significantly different than zero as highlighted in yellow.

\begin{tabular}{|l|l|l|l|l|l|l|l|}
\hline$K$ & $a$ & $w$ & $s$ & $P$ & $A$ & $w$ & $s$ \\
\hline$A$ & 1.0 & 0.57 & 0.45 & & 1.0000 & 0.0005 & 0.0089 \\
\hline$W$ & 0.57 & 1.0 & 0.66 & & 0.0005 & 1.0000 & 0.0000 \\
\hline$S$ & 0.45 & 0.66 & 1.0 & & 0.0089 & 0.0000 & 1.0000 \\
\hline
\end{tabular}

\section{Gut Inflammation}

\section{Histopathology Testing:}

All worms exposed to Styrofoam demonstrated some degree of vacuolization, with vacuoles visible within the epithelial cells lining the midgut (Figure 5), $\mathrm{p}=0.0006$. Only mild-moderate vacuolization was noted in worms exposed to bran + Styrofoam; all worms exposed to Styrofoam alone, or Styrofoam + antibiotics, demonstrated severe vacuolization.

\section{Catalase and Glutathione Testing:}

Worms exposed to Styrofoam alone, or Styrofoam + antibiotics, demonstrated decreased levels of catalase activity, a marker of oxidative stress, $\mathrm{p}=0.01$ (Figure 6). Worms exposed 
to bran + Styrofoam demonstrated increased levels of catalase activity, but these worms were taken from the group which had degraded minimal Styrofoam. A negative value was obtained for catalase activity in the Styrofoam + ciprofloxacin group. Since the catalase activity was determined by measuring the absorbance of a product of the catalase enzyme using a microplate reader, the negative value was interpreted as zero, or absent catalase activity, understanding the limitations of the technique.

There was no demonstrable change in glutathione activity within the experimental groups, $\mathrm{p}=0.87$ (Figure 7).

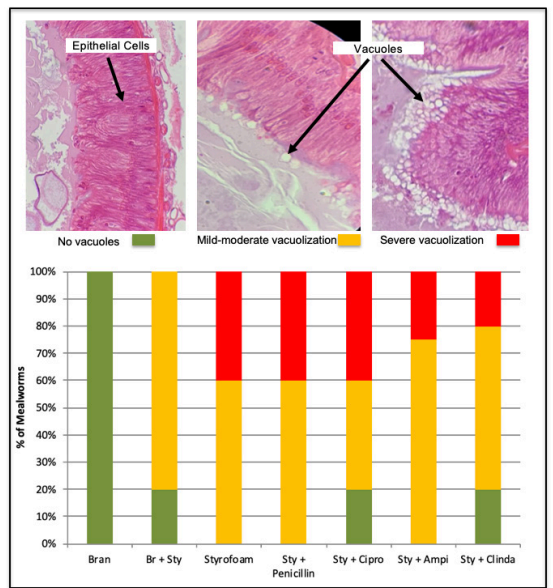

Figure 5: Vacuoles were observed in intestinal epithelial cells with Styrofoam exposure, and with increased severity with addition of antibiotics.

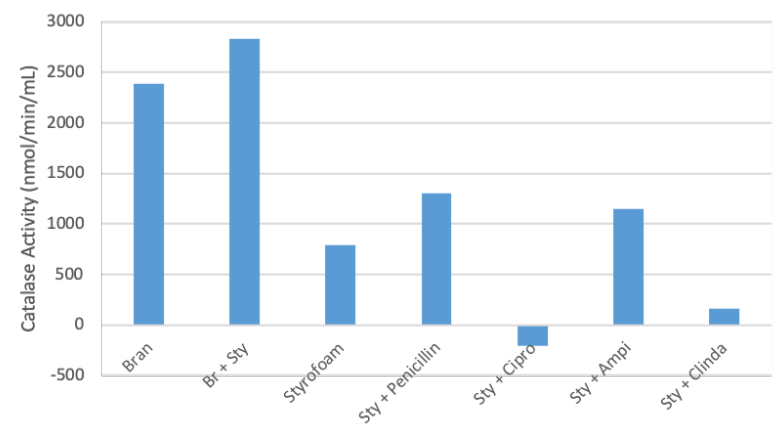

Figure 6: Gut catalase activity was decreased with Styrofoam alone, or Styrofoam + antibiotics, compared with bran or bran + Styrofoam.

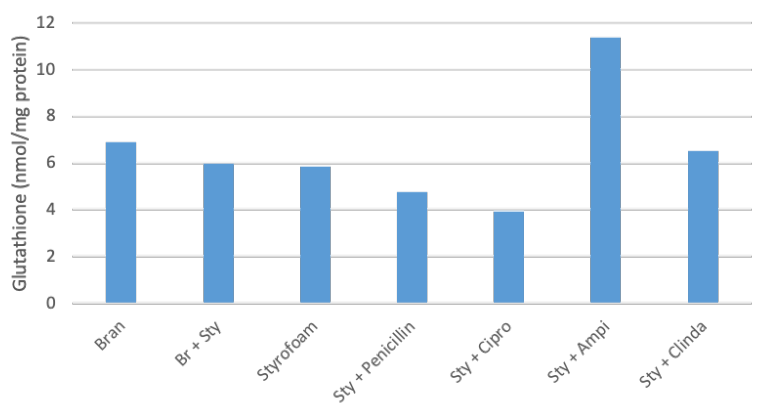

Figure 7: Gut glutathione concentration did not vary significantly among experimental groups

\section{- Conclusion}

Mealworms consumed Styrofoam without an observable impact on mortality or progression to the next phase of their life cycle (pupation), which bodes well for their resilience and their potential as a sustainable method of degrading Styrofoam. Studying the microbiome of the mealworm gut enhances our understanding of the mechanisms by which Tenebrio degrade Styrofoam. Microbial diversity appears to be substantial in maximizing Styrofoam consumption, notably so that the suppression of microbiome diversity with administration of antibiotics was associated with decreased Styrofoam digestion in this series of experiments. Spiroplasmataceae was very resistant to antibiotics, and perhaps its presence in the gut is crucial to a base threshold of Styrofoam degradation. Furthermore, bacteria that were sensitive to antibiotics may play additional essential roles in driving the consumption and processing of Styrofoam: enterobacteriaceae, enterococcaceae, lactobacillaceae, flavobacteriaceae, veillonellaceae, moraxellaceae, leptotrichiaceae, pseudomonadaceae, and cytophagaceae.

Intestinal inflammation and damage occurred after the regimented Styrofoam ingestion. Decreased midgut catalase levels with Styrofoam exposure were indicative of oxidative stress, damage done by free radicals inadequately neutralized by antioxidants. Catalase starts the decomposition of hydrogen peroxide to water and oxygen, and with decreased catalase levels, reactive oxygen species cause cellular oxidative damage and death. Midgut epithelial cell vacuolization was another manifestation of cell damage in all mealworm groups exposed to Styrofoam. Vacuoles are degradative organelles, enclosed compartments filled with water, inorganic and organic molecules, enzymes, and cellular waste. Vacuole formation in Styrofoam-fed worms may occur as cell surface proteins are degraded and processed for removal. These findings are indicative of intestinal damage and are concerning to the health of the gastrointestinal system, considering the amount of plastic and Styrofoam is ingested by humans and wildlife.

A limitation that spanned the entire study was access to finite resources, as well as the timeline. It would have been most ideal to study more worms and to repeat the microbiome, oxidative stress experiments, and histopathology studies multiple times to be able to increase sample size and achieve levels of statistical significance. With a dearth of prior studies to guide antibiotic administration, it was challenging to ensure adequate delivery of the antibiotics to the worms without overdosing. It would be optimal to determine the most effective method of giving antibiotics by conducting multiple studies evaluating different doses and routes of administration. The study design was also limited by a paucity of information about the microbiome within the mealworm gut. Furthermore, there was very little literature pertaining to the anatomy and histopathology of the mealworm midgut.

Future applications of this project include identifying specific enzymes in the bacteria of the mealworm gut and determining their specific abilities to break down Styrofoam. It would be interesting to study whether a film of the bacteria from the mealworm gut would be effective in decomposing Styrofoam. Perhaps a coating or spray of this bacterial film or enzymes from the bacteria could be a novel way of decomposing Styrofoam in the future. Additionally, it could be important to study whether ingestion of Styrofoam leads to 
additional concerning effects on the gut, including pre-cancerous changes. Lastly, a further step for this research could be to study whether humans who consume significant food and drink from Styrofoam containers demonstrate intestinal microbiome changes, and if these correlate with inflammatory bowel disease.

\section{Methods}

\section{Styrofoam-Antibiotic Experimental Setup:}

14 glass aquaria were set up with fine mesh lids in controlled conditions: 77 degrees F, 16:8 light/dark photoperiod. Mealworms were purchased from Rainbow Mealworms, Compton, CA. Seven experimental groups of mealworms were organized in duplicate (Figure 1):

a) Fed bran alone

b) Fed Styrofoam alone

c) Fed bran + Styrofoam

d) Fed bran + Styrofoam + penicillin (intended to cover gram positive bacteria)

e) Fed bran + Styrofoam + ciprofloxacin (intended to cover gram negative bacteria)

f) Fed bran + Styrofoam + ampicillin (intended to cover a broader spectrum of bacteria than penicillin, including Escherichia coli, Haemphilus influenza, Helicobacter pylori, Enterococcus faecalis, and Proteus mirabilis)

g) Fed bran + Styrofoam + clindamycin (intended to cover anaerobic bacteria).

Styrofoam blocks were weighed, and laser scanned prior to experimentation. Antibiotic - bran mixtures were prepared. Antibiotics were crushed and mixed with bran in a ratio of 30 $\mathrm{mg}$ antibiotic / $1 \mathrm{~g}$ bran based on prior work by Yang et $\mathrm{al}^{4}{ }^{4}$. A potential concern in administering the same dose of antibiotic to each group was that each drug has variable efficacy, but since there were no prior studies to guide dosage, a standard dose was chosen for all antibiotics for uniformity. ${ }^{4}$ For 7 days, in duplicate, 350 mealworms were maintained in bran alone. 115 worms were maintained in bran plus the four antibiotics, in duplicate (Figure 8). At the 7-day mark, the mealworms were removed from the bran. Mealworm intestines were excised from 5 worms from the bran and four bran + antibiotic groups for microbiome testing. The intestines were removed by dousing the worms with ethanol and cutting the inferior aspect of the worms, squeezing the intestines into sterile containers, using sterile gloves and scissors. The containers were placed on dry ice and brought for freezing at -80 degrees Celsius for later microbiome testing. For the following 20 days, in duplicate, 110 worms were placed in bran alone, 110 in bran + Styrofoam, 110 in Styrofoam, and 110 in Styrofoam + antibiotics. $500 \mathrm{mg}$ of antibiotics were crushed and sprinkled into the aquaria every three days. At the 27- day mark, all worms were removed from the aquaria. Styrofoam blocks were set aside for later weighing and laser scanning. Intestines from 5 worms from each experimental group were excised and frozen for microbiome testing. Ten mealworms from each group were brought for catalase and glutathione testing. Intestines from 5 worms from each group were prepared for histopathologic testing.

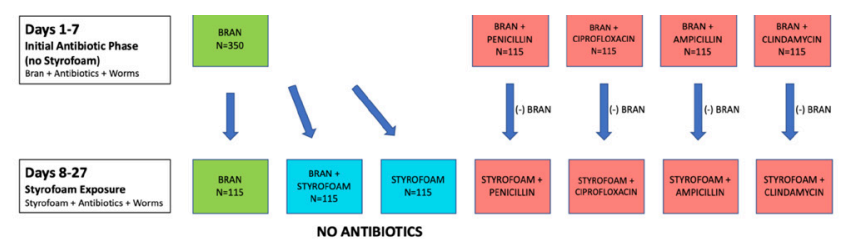

Figure 8: Styrofoam-antibiotic experimental setup.

\section{Microbial Community Analysis:}

For the microbiome testing, DNA extraction was performed using the Quick-DNA Fecal/Soil Microbe MiniPrep Kit (Zymo Research, item no. D6010). The genomic DNA needed for $16 \mathrm{~S}$ rDNA amplification of the isolated strains was extracted from cells grown in the late log phase using a conventional proteinase $\mathrm{K}$ treatment and phenol-chloroform extraction. The $16 \mathrm{~S}$ rRNA gene was sequenced following methods previously described using the $341 \mathrm{~F}$ and $806 \mathrm{R}$ universal primers to amplify the V3-V4 region. 300nt paired-end sequences were generated on the Illumina MiSeq platform. ${ }^{10}$ Reads were assembled and clustered, and a taxonomic abundance table (OTU table) was generated using the UPARSE pipeline. ${ }^{11}$ Taxonomic classifications were determined using SINTAX (2) and RDP training set v16 (with species names) (https://drive5.com/usearch/manual/sintax_downloads.html).

\section{Measures of Gut Inflammation:}

The intestines from 5 worms from each experimental group were excised and placed in Bouin's solution (Sigma-Aldrich, item no. HT10132). The guts were transversely cut and dehydrated (done by passing the tissue through solutions of increasing alcohol concentration until 100\% alcohol is reached), cleared with xylene, and embedded in paraffin wax. From these, 3 micrometer thick sections were obtained, placed on slides, de-paraffinized (with xylene solution), and rehydrated (with decreasing alcohol concentration, until 70\% alcohol is reached).

The slides were stained with hematoxylin and eosin, performed with nuclear stain (Harris hematoxylin) for 8 mins, submerged in water for 10 minutes, and counterstained with $1 \%$ alcoholic eosin, in which samples remained for 6 minutes (Sigma-Aldrich, item no. HHS16). To achieve dehydration, the reverse process was performed, passing through increasing alcohol concentrations and xylene. Slides were cover slipped and examined with light microscopy. A semi-quantitative rating was assigned to vacuolization observed in the epithelial cells.

The intestines from 10 worms from each experimental group were extracted and frozen at -80 degrees Celsius for catalase and glutathione testing. To measure catalase activity, frozen intestines were homogenized in $5 \mathrm{ml}$ of cold $50 \mathrm{mM}$ potassium phosphate buffer, $\mathrm{pH}$ 7.0, containing $1 \mathrm{mM}$ EDTA, and then centrifuged at $10,000 \times \mathrm{g}$ for $15 \mathrm{~min}$ at $4^{\circ} \mathrm{C}$. The pellet was discarded, and the resulting supernatant was used for the following steps. The absorbance of an enzyme product was read at $540 \mathrm{~nm}$ using a Bio-Rad model 550-microplate reader, according to the manufacturer's instructions (Catalase Assay Kit, Cayman Chemical, item no. 707002). One unit of catalse was defined as the amount of enzyme needed for the formation of $1.0 \mathrm{nmol}$ of formaldehyde per minute at $25^{\circ} \mathrm{C}$. 
For glutathione testing, frozen intestines were washed with chilled PBS and lysed with chilled RIPA buffer (phenylmethylsulphonyl fluoride and phosphatase inhibitor) for $30 \mathrm{~min}$. Lysates were centrifuged at $12,000 \mathrm{rpm}$ for $10 \mathrm{~min}$ at $4^{\circ} \mathrm{C}$ and the supernatant was collected for determination of glutathione activity. The concentration of protein from cell lysates was quantified using the Bradford method. ${ }^{12}$ All observations were done by using Cayman Chemical kit for glutathione according to the manufacturer's instructions (Glutathione Assay Kit, Cayman Chemical, item no. 703002).

\section{Statistical Analysis:}

One-way analysis of variance (ANOVA) testing setting $\mathrm{p}$-value significance at 0.05 was done for analyses of mealworm mortality, Styrofoam weight loss and surface damage, midgut epithelial cell vacuolization, catalase activity, and glutathione activity. Correlation coefficients were calculated to assess correlation between gut bacterial relative abundance, Styrofoam weight loss, and Styrofoam surface damage.

To quantify the correlation between the bacterial relative abundance a, Styrofoam weight loss w, and Styrofoam surface damage $s$, the number of bacteria families whose relative abundance exceeded $5 \%$ in each of the experimental categories was summed and correlated with Styrofoam weight loss and surface damage

Using MATLAB's corr function, the correlation $\mathrm{K}$ and $\mathrm{p}$-value $\mathrm{P}$ matrices for Pearson's correlation were determined as follows:

-Normalized input vector $\mathrm{X}=\left[a / \sqrt{ }\left(a^{\prime *} a\right): w / \sqrt{ }\left(w^{* *} w\right): s / \sqrt{ }\left(s^{\prime *} s\right)\right]$

-Using the following command, $[K, P]=\operatorname{corr}(X), \mathrm{K}$ is the pairwise correlation matrix and $\mathrm{P}$ is the associated $\mathrm{p}$-values for Pearson's correlation.

\section{Acknowledgements}

I would like to thank Dr.John Sullivan, the Science Department Chair at Boston College High School, and Mr. Adam Lewis, the principal, for their support and encouragement. I also would like to thank the following scientists, who so kindly helped supervise different aspects of this project: Dr. Doyle Ward of the University of Massachusetts Center for Microbiome Research in Worcester, MA, and Drs. Dipak Panigrahy and Dmitry Ratner of Beth Israel Deaconess Medical Center in Boston, MA.

\section{References}

1.Austin H., Allen M.,Donohoe B. Characterization and engineering of a plastic-degrading aromatic polyesterase.PNAS 2018. 115(19), E4350-7.

2. Yang S., Brandon A., Flanagan J., Yang J., Ning D., Cai S., Fan H., Wang Z., Ren J., Benbow E., Ren N., Waymouth R., Zhou J.,Criddle C., Wu. Biodegradation of polystyrene wastes in yellow mealworms (larvae of Tenebrio molitor Linnaeus): Factors Affecting Biodegradation Rates and the Ability of PolystyreneFed Larvae to Complete Their Life Cycle. Chemosphere 2018. 191:979-989.

3. Yang Y., Yang J., Wu W., Zhao J, Song Y., Gao L., Yang R., Jiang L. Biodegradation and Mineralization of Polystyrene by PlasticEating Mealworms: Part 1. Chemical and Physical Characterization and Isotopic Tests. Environ Sci and Tech 2015. 49:12080-12086.

4. Yang Y., Yang J., Wu W., Zhao J, Song Y., Gao L., Yang R., Jiang L. Biodegradation and Mineralization of Polystyrene by
Plastic-Eating Mealworms: Part 2. Role of gut microorganisms. Environ Sci and Tech 2015. 49:12087-93.

5. Jung J., Heo A., Park Y., Kim Y., Koh H., Par W. Gut microbiota of Tenebrio molitor and their response to environmental change. J Microbiol Biotechnol 2014. 24(7):888-987.

6. Janda J. 16s rRNA sequencing for bacterial identification in the diagnostic laboratory: pluses, perils, and pitfalls. J Clin Microbiol 2007. 2761-2764.

7. Rodriguez-Seijo A., Lourenco J., Rocha-Santos J., da Costa J., Duarte A., Vala H., Pereira R. Histopathological and molecular effects of microplastics on Eisenia Andrei Bouche. Environ Pollut 2017. 220:495-503.

8. Wan Z., Wang C., Zhou J., Shen M., Wang X., Fu Z., Jin Y. Effects of polystyrene microplastics on the composition of the microbiome and metabolism in larval zebrafish. Chemosphere 2019.https:// doi.org/10.1016/j.chemosphere.2018.11.070.

9. Lonc E., Fokt A., Andrejczal S. Histopathological effects of entomopathogenic Bacillus thuringiensis isolates on the midgut of the yellow mealworm larvae. Medycyna Wet 2007. 63(9):1049-51.

10. Kozich J., Westcott S., Baxter N., Highlander S., Schloss P. Development of a dual-index sequencing strategy and curation pipeline for analyzing amplicon sequence data on the MiSeq Illumina sequencing platform. Appl Environ Microbiol 2013. 79(17):5112-20. Epub 2013/06/25. doi: 10.1128/AEM.01043-13. PubMed PMID: 23793624; PMCID: PMC3753973.

11. Edgar RC. UPARSE: highly accurate OTU sequences from microbial amplicon reads. Nat Methods 2013. 10(10):996-8. Epub 2013/08/21. doi: 10.1038/nmeth.2604. PubMed PMID: 23955772.

12. Bradford, M. A rapid and sensitive method for the quantification of microgram quantities of protein utilizing the principle of protein-dye binding. Analytical Biochemistry 1976. 72(1-2): $248-254$.

\section{Authors}

Kieran Dunn can be found most afternoons on the Charles River, rowing with his high school crew team. He is very concerned about issues of climate change, waste minimization, and sustainability. He hopes to study engineering, environmental science, and art in college. 